\title{
TRANSURETHRAL MICROWAVE THERMOTHERAPY FOR BENIGN PROSTATIC HYPERPLASIA
}

\author{
JONATHAN N. RUBEINSTEIN, KEVIN T. MCVARY \\ Department of Urology, Feinberg Medical School, Northwestern University, Chicago, \\ Illinois, USA
}

\begin{abstract}
Transurethral resection of the prostate (TURP) remains the gold standard for treatment of benign prostatic hyperplasia (BPH). In general, while this procedure is safe, patients require a spinal, epidural, or general anesthesia and often several days of hospital stay; the potential morbidity and mortality limits the use of TURP in high-risk patients. Pharmacotherapy has been recommended as a first-line therapy for all patients with mild to moderate symptoms. Patients are oftentimes enthusiastic if they are offered a one-time method to treat lower urinary tract symptoms secondary to BPH, provided that the method offers reduced risk and allows an efficacy equal to that of medical therapy. One such method is transurethral microwave thermotherapy (TUMT). TUMT involves the insertion of a specially designed urinary catheter with a microwave antenna, which heats the prostate and destroys hyperplastic prostate tissue. TUMT allows the avoidance of general or regional anesthesia, and results in minimal blood loss and fluid absorption. In this review, the authors discussed the current indications and outcome of TUMT, including the history of the procedure, the mechanism of action, the indications for TUMT, the pre-operative considerations, the patient selection, the results in terms of efficacy, by comparing TUMT vs. Sham, TUMT vs. Alpha-blocker and TUMT vs. TURP. Finally, the complications are presented, as well as other uses and future directions of the procedure. The authors concluded that TUMT is a safe and effective minimally invasive alternative to treatment of symptomatic BPH.
\end{abstract}

Key words: prostate; prostatic hyperplasia; transurethral microwave thermotherapy; transurethral resection of prostate

Int Braz J Urol. 2003; 29: 251-263

\section{INTRODUCTION}

Transurethral resection of the prostate (TURP) remains the gold standard for treatment of benign prostatic hyperplasia (BPH). In general, while this procedure is safe, patients require a spinal, epidural, or general anesthesia and often several days of hospital stay; the potential morbidity and mortality limits the use of TURP in high-risk patients. Pharmacotherapy has been recommended as a firstline therapy for all patients with mild to moderate symptoms. Unfortunately, long-term outcomes are not fully elucidated, patients must adhere to a strict medication schedule, and outcome indicators are not reached as well or as reliably as TURP. Despite these inadequacies, patients choose medications over surgery because of the perceived reduced risk of adverse events and the desire to avoid surgery.

This trade-off of risk for efficacy is a common thread running through all elective treatments for BPH. Newer modalities have been aimed at providing alternatives to pharmacotherapy or watchful waiting. Patients are oftentimes enthusiastic if they are offered a one-time method to treat lower urinary tract symptoms secondary to $\mathrm{BPH}$, provided that the method offers reduced risk and allows an efficacy equal to that of medical therapy. One such method is transurethral microwave thermotherapy (TUMT). TUMT involves the insertion of a specially designed urinary catheter with a microwave antenna, which 
heats the prostate and destroys hyperplastic prostate tissue. TUMT allows the avoidance of general or regional anesthesia, and results in minimal blood loss and fluid absorption. Clinical trials in the United States and Europe have shown this modality to be safe and effective, with excellent symptomatic relief seen in as little as one outpatient setting using only local anesthesia. However, there is less improvement in urinary functioning seen than with TURP, and longterm follow-up data is not yet available. Clinical indications and treatment parameters for TUMT are still evolving as technology advances and more experience is gained. This manuscript summarizes current knowledge on indications and efficacy of microwave therapy of the prostate.

\section{PROCEDURE HISTORY}

In the 1980s, the use of heat to treat BPH regained clinical interest as alternatives to TURP and open prostatectomy were being explored. The modern use of microwaves has been credited to Yerushalami et al. (1). In 1982, they performed microwave therapy on a patient with prostatic adenocarcinoma (1) and later reported the therapeutic use of microwaves by the transrectal route to treat patients with $\mathrm{BPH}$ who were poor operative candidates (2).

The first machines to undergo clinical trials using hyperthermia employed a transurethral catheter in a series of ten 1-hour sessions. Software and instrumentation allowed only a limited and often interrupted delivery of energy to the prostate, with intraprostatic temperatures reaching $40-45^{\circ} \mathrm{C}$. Patients reported improved symptomatology, likely due to destruction of the alpha-adrenergic nerve fibers around the prostate, although an objective improvement of voiding parameters was not observed (3), and prostatic cells were not destroyed.

To reliably destroy cells, temperatures greater than $45^{\circ} \mathrm{C}$ were necessary, which was coined "thermotherapy" (4). Cells would slough away over a period of weeks to months. Unfortunately, the urethral pain threshold was realized to be $45^{\circ} \mathrm{C}$. The introduction of urethral cooling allowed these higher temperatures to be used. Although heat treatment pattern differed from device to device, antennae were designed to allow heat to generally follow the anatomical borders of the transition zone. Both objective and subjective measures produced significant improvement. However, patients invariably had severe prostatic edema and urinary retention, requiring the use of a urinary catheter, which became standard practice after a TUMT.

To improve outcomes even further, highenergy thermotherapy was introduced. Temperatures greater than $70^{\circ} \mathrm{C}$ were reached, causing thermoablation of prostatic tissue. Today, several different microwave devices are in use around the world, including the Targis (Urologix, Inc., Minneapolis, Minnesota, USA), Prostatron (Technomed Medical System, Lyons, France), Prostalund (Lund Instruments AB, Lund, Sweden), Prostcare (Bruker Medical, Wissembourg, France), Urowave (Dornier MedTech America, Kennesaw, Georgia, USA), PRIMUS U+R (Tecnomatix, Monheim, Germany), and the LEO Microthermer (Laser Electro Optics, London, UK).

\section{MECHANISM OF ACTION}

TUMT uses an external power source creating microwaves at a frequency of 915-1296 MHz. Tissue penetration of microwaves leads to electromagnetic oscillations of free charges and the polarization of small molecules, such as water, resulting in the release of kinetic energy, which increases the temperature of the tissue. Cell necrosis, vascular injury, and apoptosis ensue. Urethral cooling in part protects the prostatic urothelium from these effects.

\section{INDICATIONS FOR TUMT}

It is widely agreed upon that patients with mild to moderate symptoms should be treated with medical therapy initially, reserving invasive therapies for those with more severe symptoms, those failing medical management, and for patients choosing invasive therapy over medical management. As the criterion standard for invasive therapy, TURP is offered to most patients. Initially, only patients with severe symptoms who were poor anesthetic risks were 
offered TUMT. Indications have now been expanded to include patients who prefer and outpatient setting rather than for a hospital stay due to the ease of use, minimal anesthesia requirement, and potentially rapid recovery.

\section{PRE-OPERATIVE CONSIDERATIONS}

\section{Patient Selection}

A thorough clinical evaluation is vital to assess the presence and degree of voiding dysfunction and/or the role played by BPH. The patient's past urologic history along with surgical risks and concomitant medical problems need to be evaluated. A history of sexually transmitted diseases, kidney stones, trauma, previous catheterizations, genitourinary cancer, renal insufficiency, neurologic disease, and neurogenic bladder may influence the treatment options. Medical conditions that may influence bladder functioning mimicking BPH-type symptoms include diabetes and neurological diseases. Surgical risks predominantly are due to renal failure, coronary artery disease, and cerebrovascular disease. Medicines containing alpha-sympathomimetics, such over-the-counter cold remedies, enhance bladder outlet obstruction. A family history should focus on a history of urologic cancer, and a social history should focus on risks for cancer such as a smoking history and occupational exposure.

The physical examination should be systematic and meticulous, focusing on the presence or absence of distended bladder, urethral stenosis, meatal stenosis, and anal area and rectal tone. The prostate is evaluated for its size, the presence or absence of nodularity, laterality, consistency, and landmarks.

\section{Laboratory Studies}

A hemoglobin determination and platelet count are not specifically required prior to TUMT unless the patient has a history of anemia, coagulation disorder, or as suggested by the history and physical examination. Patients with a renal insufficiency (baseline creatinine greater than $1.7 \mathrm{mg} / \mathrm{dL}$ ) due to post-renal obstruction may benefit more from a more definitive procedure such as TURP. A determination of serum PSA level may be important in the evaluation of the absence of prostate cancer. Unlike TURP, no specimen is submitted to pathology after TUMT. Even with a normal PSA and negative biopsies, patients are at risk for prostate cancer, which may be observed on the chips. If clinically suggested, transrectal biopsies should be performed, as alternative therapies may be suggested. Studies using baseline PSA as a predictive factor for TUMT success are varied. In addition, all patients should have documented negative culture before any urethral instrumentation to decrease the risk of urosepsis.

\section{Imaging Studies}

A transrectal ultrasound (TRUS) is suggested before performing TUMT, for patients with prostate volumes estimated to be less than $25 \mathrm{cc}$ or greater than $100 \mathrm{cc}$ respond poorly to TUMT. Renal ultrasound should be performed if there is a history of urinary retention, have evidence of renal insufficiency, or there is hematuria.

\section{Diagnostic studies}

A cystourethroscopy is mandatory to evaluate the urethra for stricture disease, prostatic length, and degree of prostatic hypertrophy. Patients with lateral lobe hypertrophy respond much better to TUMT than those with middle lobe hypertrophy or a median bar, as marked middle lobe enlargement distorts the heating pattern. The urethra and bladder urothelium should also be evaluated for evidence of tumors and stones. The location of the ureteral orifices should be noted.

The maximal voiding rate $(\mathrm{Qmax})$ is a noninvasive but nonspecific electronic recording of urinary flow rate. This is mainly used to monitor response to treatment, although patients may have a weak urinary stream due to an inadequate detrusor contraction rather than bladder outlet obstruction. An adult man without evidence of obstruction should have an average velocity of $12 \mathrm{cc} / \mathrm{sec}$ and an average peak velocity of $20 \mathrm{cc} / \mathrm{sec}$. The prediction capacity of Qmax for outcome remains a matter of debate.

Symptom indices (including the AUA score, IPSS, Madsen quality of life, and Boyarsky) are available and are used to confirm the components of 
the patient's history, evaluate and quantify the patient's response to treatment, and to compare the results of research protocols. Studies have failed to document a strong correlation between symptom scores and physiologic changes due to $\mathrm{BPH}$.

A post-void residual (PVR) volume is often measured. While this does not predict surgical outcome, it may be used to determine how closely patients need to be followed. Patients with high PVRs have slightly higher rates of failure of watchful waiting and are at an increased risk of complications such as urinary tract infections and renal failure. A pressure-flow study should be performed in patients whose voiding velocity and PVR measurements are not sufficient to determine the presence or absence of bladder outlet obstruction or poor detrusor contraction. This is the study of choice in patients with a history of neurologic disease or other problems that affect the bladder and may confound the diagnosis, and for patients with normal flow rates but bothersome symptoms.

A cystometrogram $(\mathrm{CMG})$ using either gas or liquid may be of value for patients with known or suspected neurological impairment. While it is less specific than pressure-flow studies, it allows the evaluation of bladder capacity, evidence of uninhibited detrusor contractions, and an estimation of bladder compliance. Patients who have adequate bladder contractions have better outcomes after TUMT. A urethral pressure profile measures pressures along the length of the urethra. In patients with known or suspected urethral obstruction, this test helps determine the location of the lesion. Finally, videourodynamics may be useful for patients with complex causes of outlet obstruction or for cases where the sites of obstruction need to be identified. Patients with neurogenic bladders may benefit as well as patients undergoing an evaluation for incontinence.

\section{HISTOLOGICAL FINDINGS AFTER TUMT}

Bostwick \& Larson (5) studied the results of TUMT on 3 dogs, showing that there was periurethral coagulative necrosis acutely, which began to resolve within 18 days. The capsule and urethra were deemed to be intact. Mauroy et al. (6) reported maximal coagulative necrosis at 8 days. Bdesha et al. (7) reported a significant decrease in alpha-1 adrenoreceptors after TUMT. Khair et al. (8) performed radical prostatectomies on at 7 days and 1 year later after TUMT, reporting that hemorrhagic necrosis and devitalized tissues without inflammation was observed in benign, stromal, and cancerous areas early on, with a mean volume of necrosis being 8.8 cc (average $22 \%$ of the tissue), but that only nonspecific chronic inflammation and a desquamous metaplasia with evidence of periurethral fibrosis remained at one year. The mean volume of necrosis remaining was $0.2 \mathrm{cc}$, which was less than $1 \%$, implying that cells were sloughed away.

\section{CONTRAINDICATIONS}

All patients undergoing transurethral procedures must have a documented sterile urine culture and must be evaluated for prostate or urothelial cancer if clinically suspected. Patients with neurogenic bladder voiding dysfunction should have their underlying neurogenic problem evaluated and treated.

Contraindications specific to TUMT are evolving as the technology changes and outcomes are studied further. Patients with a history of TURP or pelvic trauma should not undergo TUMT because of potential alterations in pelvic anatomy. Patients with glands that are smaller than $25 \mathrm{gm}$. or a prostatic urethral length of less than $3 \mathrm{~cm}$ respond poorly to TUMT, as do patients with glands greater than 100 gm or patients with a prominent median bar.

Other contraindications include patients with penile prosthesis, severe urethral stricture disease, Leriche syndrome/severe peripheral vascular disease, or an artificial urinary sphincter. Patients with pacemakers and defibrillators need clearance from their cardiologist concerning turning their pacemakers off during therapy.

Hip replacement is no longer a contraindication. Acute urinary retention was previously thought to be a contraindication to TUMT; however, high-energy TUMT has shown to be promising in this population, although efficacy has yet to be determined. Those presenting in retention 
tend to be ill with greater co-morbidities; thus, they might benefit from the less invasive nature of TUMT.

\section{PREOPERATIVE DETAILS}

In preparation for TUMT, patients need to be counseled about the risks, benefits, alternatives, and expectations of the therapy. Patients who have a urinary catheter in place or had recent urinary tract manipulation should be placed on appropriate antibiotics. The patient should have nothing by mouth for 6 hours prior to the therapy. An appropriate oral analgesic (such as ibuprofen, ketorolac, or morphine) and an anxiolytic (benzodiazepine) may be administered prior to the procedure.

The patient is brought to the therapy suite and asked to void to completion. The bladder is emptied by straight catheterization, and $40 \mathrm{cc}$ of sterile water is placed within the bladder. $10-20 \mathrm{cc}$ of $1-2 \%$ Xylocaine gel is inserted within the urethra for anesthesia. The treatment catheter is then placed within the urethra, confirmed by return of the sterile water and by transabdominal or transrectal ultrasound, and the balloon is inflated. This catheter has a curved tip, a temperature sensor, and a microwave unit near the tip. The distal ports include those for balloon inflation, urine drainage, coolant, microwave cable, and fiber optic connector. The rectal probe (if used) is inserted and continuously monitors the rectal temperature.

\section{POSTOPERATIVE DETAILS}

Out patients and practice are such that all patients are catheterized following TUMT. They return to the clinic for a trial of decatheterization, varying by the protocol used. As prostatic edema is nearly universal after microwave therapy, there are a high number of patients that fail the initial decatheterization trial if performed too early.

Post-treatment convalescence is relatively rapid, with most patients able to void in less than 3 days at home and a mean recovery time of 5 days at home. Catheterization is required in approximately $60 \%$ of patients after low-energy Prostatron 2.0 and $100 \%$ of the high-energy Prostatron 3.5, and often this is extended for over 2 weeks. Patients are typically catheterized for a minimum of 2 days after Targis thermotherpay. Patients with larger prostates are more prone to catheterization because of increased edema. The slow process of improvement is characteristic of high-energy transurethral microwave thermotherapy. Coagulated tissue must be absorbed and the treated area must be reorganized before sufficient voiding is achieved. Patients may notice an improvement over a period of many months. Patients are advised to watch for the inability to void, painful voiding, high fevers, abdominal pain, or other problems.

Some patients are maintained on alphablockade for a period of time after TUMT. Studies have shown that these patients have improved symptomatology early on over those not on alphablockade (9), and also have a lower incidence of retention. Another option includes the placement of a temporary prostatic bridge catheter, which is effective and well-tolerated option that provides a better immediate peak flow, IPSS, and quality of life compared to no stent if the patient has adequate detrusor contraction $(10,11)$.

\section{RESULTS}

\section{Efficacy}

Microwave thermotherapy has been around long enough for both short and medium-term results to be reported (Table-1). In general, patients have noticeable symptomatic improvements as shown by a reduction in symptom scores, although complementary objective improvements have not occurred as regularly (12-26). Long-term follow-up in patients undergoing low-power TUMT reveals subjective symptom score improvement, although for only limited duration in most cases; in the long-term, only a minority of patients showed benefit from treatment. Retreatment rates are substantial, reportedly $57-84 \%$ by 5 years. Using the low-energy Prostatron Prostasoft 2.0, Ohigashi et al. (25) reported a stimated of $67 \%$ retreatment rate with 5 years with only $11 \%$ "satisfied" with treatment, while Tsai et al. (20) reported an overall retreatment rate of $84.4 \%$ in 5 years using Prostcare. In both of these studies, patients with high peak flow rates (i.e. $>6.5 \mathrm{cc} / \mathrm{sec}$ ) 


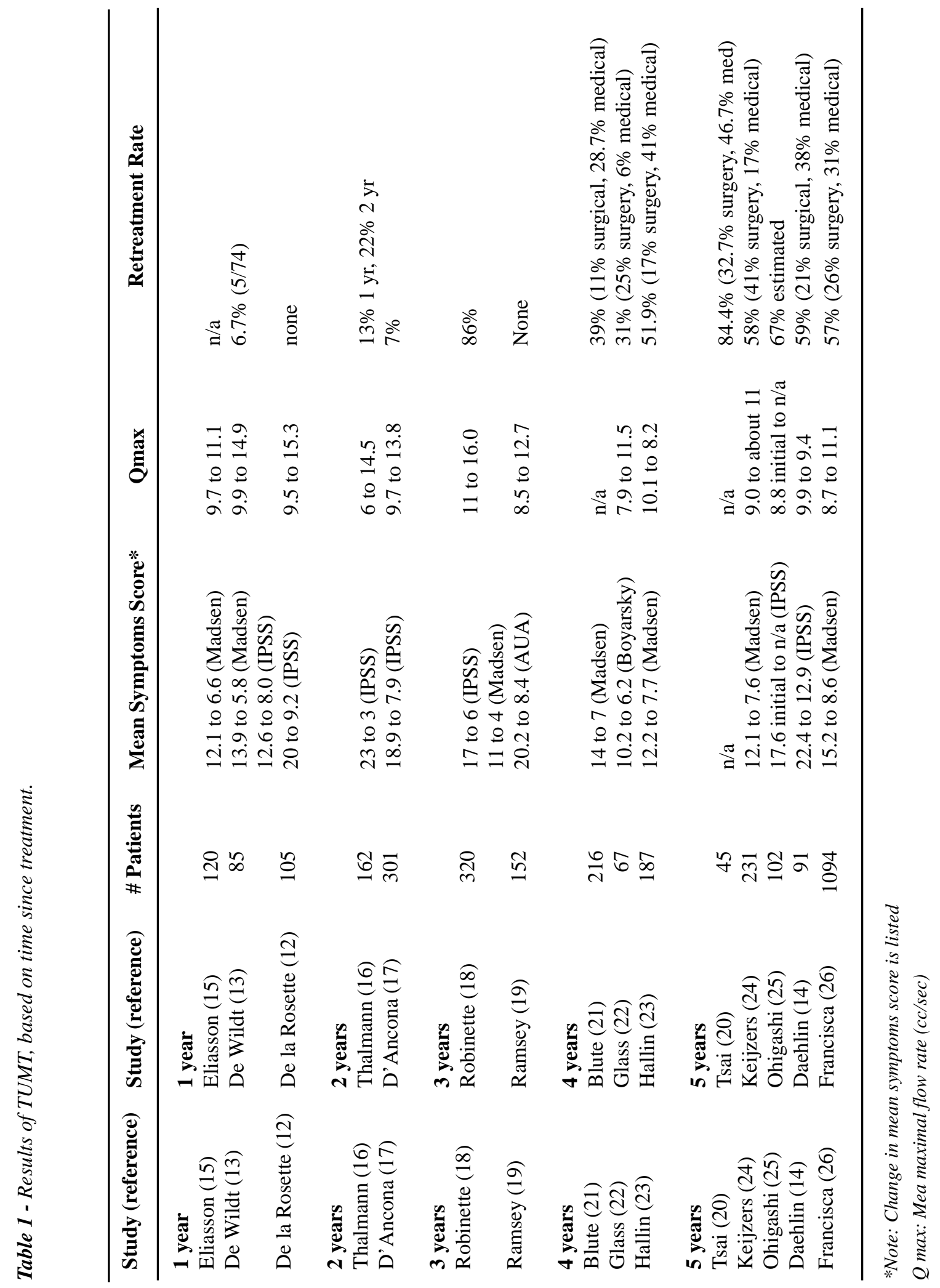


and short urethral lengths (i.e. $<40 \mathrm{~mm}$ ) had a lower risk of receiving additional treatments after TUMT. Interestingly, in both studies, older patients (i.e. > 64 years old) similarly had a decreased rate of retreatment. Keijzers et al. (24) similarly reported a decreased risk of retreatment in patients with pretreatment maximal urinary flow greater than $10 \mathrm{cc} /$ sec, Madsen symptom score less than 15, post-void residual urine less than $100 \mathrm{cc}$, and age greater than 65 years.

In comparison, higher energy protocols appear to result in symptomatic improvement similar to that of lower energy protocols yet the improvement in uroflowometry is much more pronounced. This appears to be in trade-off for greater irritative symptomatology and longer duration of catheterization (12). In patients with good initial responses to treatment, which is achieved in approximately $80 \%$, TUMT provides better long-term subjective and objective results. Improved urinary flow, decreased postvoid residual urine volume and urodynamic parameters appear to remain stable at 2 years. In a two-year follow-up using the Targis, Thalmann et al. (16) reported that only $22 \%$ required additional treatments. In the remaining patients, the IPSS decreased from an average of 23 to 3 at 6 months, while the maximal flow rate increased from $6 \mathrm{ml} / \mathrm{sec}$ to 14.5 at 6 months. Both results remained durable through 24 months. Post-void residual volumes also appear to be markedly decreased after high-energy TUMT, as well as demonstrable decreases in detrusor opening pressures and median detrusor pressure at maximum flow rate (17). De la Rosette et al. (12) reported that 6 months after treatment with the Prostasoft 3.5, patients had an average decrease in the IPSS from 20 to 9.3, an increase in flow rate from $9.4 \mathrm{cc} / \mathrm{sec}$ to $14.6 \mathrm{cc} / \mathrm{sec}$, an average catheter time of 18 days, and no serious complications.

\section{TUMT vs. Sham}

Several small studies of randomized controlled trials comparing TUMT versus sham treatments (Table-2) are available, and generally show improvement of TUMT over sham. In the largest study (220 men), Roehrborn et al. (27) reported a decrease in AUA symptom score from 23.6 to 12.7 after TUMT, compared to 23.9 to 18 in the sham group, while the Qmax increased from 7.7 to $10 \mathrm{cc} / \mathrm{sec}$ and 8.1 to 9.8 $\mathrm{cc} / \mathrm{sec}$ in each group respectively. Blute et al. (28) and Larson et al. (29) similarly reported a significant improvement in the TUMT group compared to sham treatment using the Prostasoft 2.0 and Targis respectively. After reporting their results, de Wildt et al. (30) commented that "a beneficial effect of heating at thermotherapy temperatures is conclusive and further sham studies would be unethical". In contrast, neither Nawrocki et al. (31) nor Mulvin et al. (32) showed any significant improvement of TUMT over sham treatment in their studies using the Prostatron 2.0 and Targis respectively.

\section{TUMT vs. Alpha-blocker}

When compared to alpha-blockade, TUMT is associated with an initially poorer outcome but eventually better result. Djavan et al. $(33,34)$ prospectively studied 51 patients undergoing highenergy TUMT and 52 patients on terazosin therapy. Terazosin appeared to have a more rapid onset of action, and had its maximal effects observed at 6 weeks of therapy. In contrast, the maximal effect of TUMT was not observed until after 6 months of therapy. Those patients on terazosin therapy similarly were reported to have a more favorable change in the IPSS, peak flow, and quality of life at 2 weeks compared to TUMT. However, by 1,4 , and 6 months, while there was a significant improvement in both cohorts, all patients were more improved with TUMT. At 18 months, the IPSS, Qmax, and quality of life was $35 \%, 22 \%$, and $43 \%$ better in the TUMT group. Alpha-blocker therapy was associated with more adverse events (17/52) compared to TUMT (7/51). Patients on alpha-blockade complained of dizziness, asthenia, headaches, and lack of effectiveness, prompting discontinuation in $11.5 \%$ of patients. In addition, there was a sevenfold greater treatment failure rate seen in the terazosin group.

\section{TUMT vs. TURP}

There have been several trials comparing TUMT and TURP (Table-2). In generally, these studies have shown TUMT to be an effective modality, but in no way duplicated the results of TURP in terms 


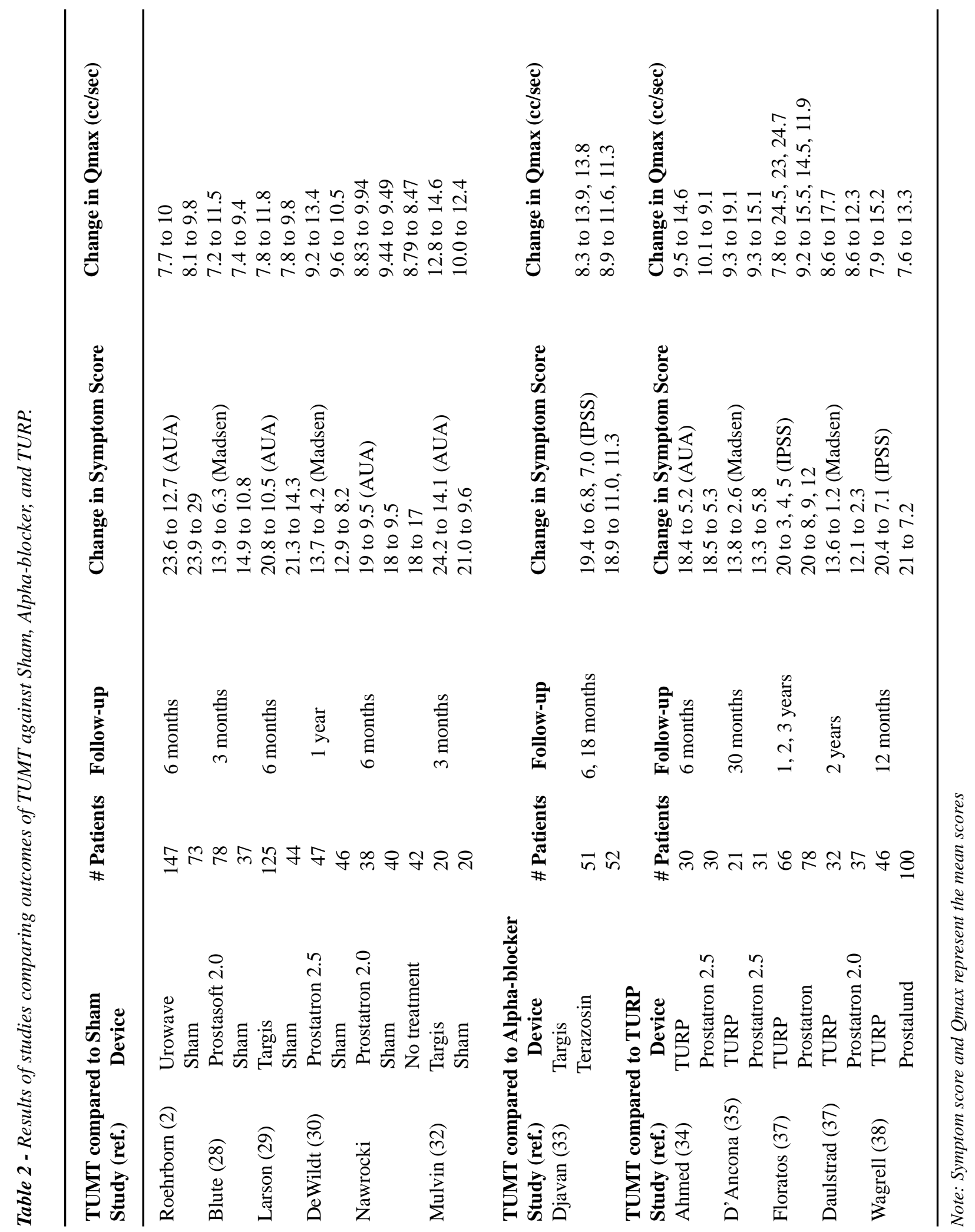


of objective or subjective parameters. For example, Ahmed et al. (35) reported that $60 \%$ of a cohort of 30 patients reported symptomatic improvement after TUMT, but none had objective relief of obstruction. In comparison, $100 \%$ of the patients in the TURP cohort showed relief of obstruction. At a longer follow-up of 2.5 years, d'Ancona et al. (36) reported that TURP performed better than TUMT using both subjective (Madsen score improvement of $76 \%$ after TURP compared to $56 \%$ after TUMT) and objective (flow rates increased $105 \%$ after TURP compared to $62 \%$ after TUMT) parameters. Floratos et al. (37) showed that these differences became more pronounced with time, as patients undergoing TURP continued to improve in both subjective and objective parameters after 1, 2, and 3 years, which was not the case in the TUMT group. Further procedures were required in $19.8 \%$ of patients undergoing TUMT, compared to $12.9 \%$ of the TURP group at 33 months.

In an attempt to bridge this gap, Wagrell et al. (38) compared patients undergoing TURP to those undergoing TUMT with the ProstaLund Feedback Treatment, which provides a feedback system based on intraprostatic temperatures during treatment. In 133 patients with a minimum of 12 months follow-up, good outcomes were reported in $82 \%$ and $86 \%$ of the patients in the TUMT and TURP respectively, and symptoms score and maximal flow rate improvements appeared to be similar. Whether or not this remains durable in the long-term remains to be seen.

Adverse effects of the 2 modalities vary. In general, patients requiring further procedures after TUMT were due to therapy failure, while after TURP was due to complications. Mild and moderate adverse events were more common in the TUMT group, but serious adverse events were more common (17\% vs. $2 \%$ ) in the TURP group. The complication rate of TURP in these series varied, and was generally due to bleeding and clot retention, urinary retention, urinary tract infections, urethral strictures, and retrograde ejaculation. In contrast, patients undergoing TUMT were at higher risk for severe urinary urgency, urinary retention, and many required secondary procedures. The average catheter time was 3 days with TURP, 1-3 days with low-energy TUMT and 3-14 days using high energy TUMT.
The reported rate of changes in sexual function is $17 \%$ with TUMT compared to $36 \%$ with TURP, which is higher in older men. One of the most common adverse effects is retrograde ejaculation. This is reportedly observed in 48-90\% of patients after TURP and $0-28 \%$ of patients after TUMT, although even alpha-blockers are associated with a risk of retrograde ejaculation. If explained prior to therapy, this complaint is generally not a concern to most patients.

Arai et al. (39) reported a $26.5 \%$ rate of erectile dysfunction for TURP and $18.2 \%$ with TUMT using a high-energy protocol.Overall, satisfaction with sex life seems to be higher in patients who have had TUMT than in patients who have had TURP, with patients undergoing microwave thermotherapy reported as being "very satisfied" in 55\% versus $21 \%$ of TURP. However, only $27 \%$ of this population is satisfied with their urinary flow after TUMT compared to $74 \%$ of patients who are satisfied after TURP.

\section{TUMT in patients in urinary retention}

Because patients presenting with urinary retention generally are older, have a larger prostate volume, and have more renal insufficiency, they are at increase anesthetic risk, increased risk for secondary procedures, and risk for bleeding. In the past, TUMT was thought to be contraindicated because of a high failure rate. However, with the advent of high-energy TUMT, patients are now offered this less-invasive therapy. Djavan et al. reported a $94 \%$ success rate at 12 weeks in 31 patients presenting in retention (40), although the 1-year retreatment rate is estimated to be $25 \%$ (41). Schelin reported that $80 \%$ of their cohort was relieved of an indwelling catheter after TUMT, and those who failed all had large median lobes or protruding lateral lobes into the bladder (42). Robinette et al. (18) reported that 44 of 60 patients in retention were able to void spontaneously at 6 months after therapy with the Prostatron 2.0 or 2.5 , as were 32 of 35 followed at 12 months.

\section{COMPLICATIONS}

During the procedure, patients commonly experience mild perineal warmth, mild pain, and a 
sense of urinary urgency. However, only $5 \%$ of patients reported their pain as being severe during Targis therapy. Despite this, more than one half of these patients required substantial oral analgesics during treatment. Higher energy protocols appear to have a slightly higher level of initial pain due to the initial higher power, which appears to resolve and return to the same level of comfort as the lower-energy protocols soon into treatment.

Reports of complications vary, and range from 0 to $38 \%$, based on the study and the investigators' criteria for complications. For example, Ohigashi et al. reported no serious side-effects in 91 patients treated with low-power over 5-year followup (25). Others report complications including acute urinary incontinence, urinary tract infection, and urinary retention. The risk for urinary tract infections rises with each day of catheterization. In addition, the necrotic tissue that remains in the prostatic fossa after TUMT may increase the risk of colonization and infection. Treatment morbidity of higher energy protocols is moderate and consists mainly of the need for catheterization and a higher percentage of retrograde ejaculation (17):

Erectile dysfunction after TUMT is rare if a patient is previously normal, but is commonly observed in patients with prior erectile difficulties. Although causes have not been fully elucidated, psychogenic factors, bladder neck trauma, and neurogenic voiding dysfunction probably play a role. Lower-energy TUMT protocols have a lower incidence of erectile dysfunction compared to higher-energy protocols but at the expense of better urinary results. Francisca et al. (43) reported no change in sexual performance after low-energy TUMT when compared to a sham procedure in 147 patients.

A variety of other rare but reported complications following TUMT occur. This includes, but is not limited to, urethrorectal fistula (44), bladder perforation, and improper catheter placement. An emphysematous prostatic abscess (45) has been reported after low-energy TUMT in a 55-year old man with diabetes mellitus and cirrhosis. Proper intratreatment physician and nursing observation are vital to decrease these risks.

\section{OTHER USES AND FUTURE DIRECTIONS}

Microwave therapy may be of value to treat other types of prostate pathology. For example, several investigators are using TUMT to treat chronic prostatitis (46). Microwave therapy is known to be lethal to many microorganisms because microwaves are used to sterilize urinary catheters and surgical scalpels. Patients with nonbacterial prostatitis who are non-responders to traditional therapy may benefit from TUMT. Early results are promising, with a $25 \%$ complete and sustained improvement and 50\% mild improvement in a group of 45 patients using TUMT.

In the future, because of the risk factors for patients with symptomatic BPH, patients may be better stratified in order to determine the optimal choice of therapies (i.e., pharmacotherapy vs. TURP vs. TUMT vs. other method). Responders and nonresponders may be differentiated better by prostatic biopsy. The optimal combination of preoperative medicines may allow for an increase in comfort. The optimal time and energy requirements for therapy will decrease morbidity. The long-term results of the balance between patient tolerability and efficacy need to be evaluated adequately in a controlled setting.

Currently underway is the MIST trial, which is a prospective, National Institute of Healthsponsored trial comparing the outcomes of transurethral needle ablation - TUNA, TURP, or combination medical therapy (alpha-blocker and 5alpha reductase inhibitor). The end-points and goals of this study are to evaluate the perioperative morbidity (i.e. pain), complications, and long-term outcomes of these three options in the treatment of $\mathrm{BPH}$.

\section{CONCLUSIONS}

TUMT is a safe and effective minimally invasive alternative to treatment of symptomatic BPH. TUMT can be performed in a 1- to 2-hour office visit without IV sedation. This is a good alternative for patients who are at high surgical and anesthetic risk. It is not effective for patients with a large median lobe or a very large prostate and results in less urinary flow patterns than TURP. 
Enthusiastic reassessment of procedures that may reduce local and overall morbidity and maintain or improve immediate and long-term physiologic results is understandable and laudable. Currently, the limited number of patients, evolving selection and technical approaches, hampers assessment of these efforts and the limited period and nature of the followup information provided. In summary, this minimally invasive therapy appears to balance efficacy against tolerability, and this balance might be tenuous for patients in the long term.

\section{REFERENCES}

1. Yerushalmi A, Servadio C, Leib Z, Fishelovitz Y, Rokowsky E, Stein JA: Local hyperthermia for the treatment of carcinoma of the prostate: a preliminary report. Prostate 1982; 3: 623-30.

2. Yerushalmi A, Fishelovitz Y, Singer D: Localized deep microwave hyperthermia in the treatment of poor operative risk patients with benign prostatic hyperplasia. J Urol. 1985; 133: 873-6.

3. Sapozink MD, Boyd SD, Astrahan MA: Transurethral hyperthermia for benign prostatic hyperplasia: preliminary clinical results. J Urol. 1990; 143: 944-9.

4. Brehmer M, Svensson I: Heat-induced apoptosis in human prostatic stromal cells. BJU Int. 2000; 85: 535-41.

5. Bostwick DG, Larson TR: Transurethral microwave thermotherapy. Pathologic findings in canine prostates. Prostate 1995; 26: 116-22.

6. Mauroy B, Chive M, Stefaniak X, Demetriou D, Prevost B, Hattab B, et al.: Study of the effects of thermotherapy in benign prostatic hyperplasia. Eur Urol. 1992; 32: 198-208.

7. Bdesha AS, Schachter M, Sever P, Witherow RO: Radioligand-binding analysis of human prostatic alpha1 adrenoreceptor density following transurethral microwave therapy. Br J Urol. 1996; 78: 886-92.

8. Khair AA, Pacelli A, Iczkowski KA: Does transurethral microwave thermotherapy have a different effect on prostate cancer than on benign or hyperplastic tissue? Urology 1999; 54: 67-72.

9. Djavan B, Shariat S, Fakhari M: Neoadjuvant and adjuvant alpha-blockade improves early results of highenergy transurethral microwave thermotherapy for lower urinary tract symptoms of benign prostatic hyperplasia: a randomized, prospective clinical trial. Urology 1999; 53: 251-9.
10. Djavan B, Fakhari M, Shariat S, Ghawidel K, Marberger M: A novel intraurethral prostatic bridge catheter for prevention of temporary prostatic obstruction following High-Energy transurethral microwave thermotherapy in patients with benign prostatic hyperplasia. J Urol. 1999; 161: 144-51.

11. Djavan B, Ghawidel K, Basharkhah A, Hruby S, Bursa $\mathrm{B}$, Marberger M: Temporary intraurethral prostatic bridge catheter compared to neoadjuvant and adjuvant alpha-blockade to improve early results of high-energy transurethral microwave thermotherapy. Urology. 1999; 54: 73-80.

12. de la Rosette JJ, Francisca EA, Kortmann BB: Clinical efficacy of a new 30-min algorithm for transurethral microwave thermotherapy: initial results. BJU Int. 2000; 86: 47-51.

13. de Wildt MJAM, Debruyne MH, de la Rosette JJMCH: High-energy transurethral microwave thermotherapy: a thermoablative treatment for benign prostatic obstruction. Urology. 1996; 48: 416-23.

14. Daehlin L, Frugard J: Transurethral microwave thermotherapy in the management of lower urinary tract symptoms from benign prostatic obstruction: followup after five years. Scand J Urol Nephrol. 2000; 34: 304-8.

15. Eliasson TU, Abramsson LB, Pettersson GT: Responders and non-responders to treatment of benign prostatic hyperplasia with transurethral microwave thermotherapy. Scand J Urol Nephrol. 1995; 24: 18391.

16. Thalmann GN, Mattei A, Treuthardt C, Burkhard FC, Studer UE: Transurethral microwave therapy in 200 patients with a minimum followup of 2 years: urodynamic and clinical results. J Urol. 2002; 167: 2496-501.

17. d'Ancona FC, Francisca EA, Debruyne FJM, de la Rosette JJ: High-energy transurethral microwave thermotherapy for men with lower urinary tract symptoms. J Endourol. 1997; 11: 285-9.

18. Robinette MA, Honey JD, Buckley RJ, Forder WC, Spevack LM, DiConstanzo JA, et al.: Results of transurethral microwave thermotherapy (TUMT) in patients presenting with: 1. Symptomatic BPH or 2 . Urinary retention. J Urol. 1997; 157 (suppl), 375A.

19. Ramsey EW, Miller PD, Parsons K: Durability of results with transurethral microwave thermotherapy for symptomatic benign prostatic hyperplasia (BPH) using the Targis (T3) system. J Urol. 1998; 159: 304A.

20. Tsai YS, Lin JS, Tong YC, Tzai TS, Yang WH, Chang $\mathrm{CC}$, et al.: Transurethral microwave thermotherapy for 
symptomatic benign prostatic hyperplasia: long-term durability with Prostcare. Eur Urol. 2001; 39: 688-92.

21. Blute ML, Hanson K, Lynch J, Tomera K, Cope P, Sankey N, et al.: United States Prostatron TUMT study - 4 year follow-up and quality of life. J Urol. 1996; 155: 403 A, Abstract 370.

22. Glass JM, Bdesha AS, Witherow RO: Microwave thermotherapy: A long-term follow-up of 67 patients from a single center. Brit J Urol. 1988; 81: 377-82.

23. Hallin A, Berlin T: Transurethral microwave thermotherapy for benign prostatic hyperplasia: clinical outcome after 4 years. J Urol. 1998; 159: 459-64.

24. Keijzers GH, Francisca EA, d'Ancona FC, Kiemeney LA, Debruyne FM, de la Rosette JJ: Long-term results of lower energy transurethral microwave thermotherapy. J Urol. 1998; 159: 1966-72.

25. Ohigashi T, Babsa S, Ohki T, Nakashima J, Murai M: Long-term effects of transurethral microwave thermotherpay. Int J Urol. 2002; 9: 141-5.

26. Francisca EA, Keijzers GB, d'Ancona FC, Debruyne FM, de la Rosette JJ: Lower energy thermotherapy in the treatment of benign prostatic hypertrophy: longterm follow-up results of a multicenter international study. World J Urol. 1999; 17: 279-84.

27. Roehrborn CG, Preminger G, Newhall P: Microwave thermotherapy for benign prostatic hyperplasia with the Dornier Urowave: results of a randomized, doubleblind, multicenter, sham-controlled trial. Urology 1998; 51: 19-28.

28. Blute ML, Patterson DE, Segura JW, Tomera KM, Hellerstein DK: Transurethral microwave thermotherapy vs. sham treatment. Double-blind randomized study. J Endourol. 1996; 10: 565-73.

29. Larson TR, Blute ML, Bruskewitz RC, Mayer RD, Ugarte RR, Utz WJ: A high-efficiency microwave thermoablation system for the treatment of benign prostatic hyperplasia. Results of a randomized, shamcontrolled, prospective, double-blind, multicenter trial. Urology 1998; 51: 731-42.

30. De Wildt MJ, Hubregtse M, Ogden C, Carter SS, Debruyne FM, de la Rosette JJ: a 12-month study of the placebo effect in transurethral microwave thermotherapy. Brit J Urol. 1996; 77: 221-7.

31. Nawrocki JD, Bell TJ, Lawrence WT, Ward JP: A randomized controlled trial of transurethral microwave thermotherapy. Br J Urol. 1997; 79(3): 389-93.

32. Mulvin M, Creagh T, Kelly D, Smith J, Quinlan D, Fitzpatrick J: Transurethral microwave thermotherapy vs. transurethral catheter therapy for benign prostatic hyperplasia. Eur Urol. 1994; 26: 6-9.
33. Djavan B, Roehrborn CG, Shariat S: Prospective randomized comparison of high energy transurethral microwave thermotherapy versus alpha-blocker treatment of patients with benign prostatic hyperplasia. J Urol. 1999; 161: 139-43.

34. Djavan B, Seitz, C, Roehrborn CG, Remzi M, Fakhari M, Waldert M, et al.: Trargeted transurethral microwave thermotherapy versus alpha-blockade in benign prostatic hyperplasia: outcomes at 18 months. Urology 2001; 57: 66-70.

35. Ahmed M, Bell T, Lawrence WT, Ward JP, Watson GM: Transurethral microwave thermotherapy (Prostatron version 2.5) compared to transurethral resection of the prostate for the treatment of benign prostatic hyperplasic: a randomized, controlled, parallel study. Br J Urol. 1997; 79: 181.

36. d'Ancona FC, Francisca EA, Witjes EP, Welling L, Debruyne FM, de la Rosette JJ: Transurethral resection of the prostate versus high-energy thermotherapy of the prostate in patients with benign prostatic hyperplasia: long-term results. Br J Urol. 1998; 81: 259.

37. Floratos DL, Kiemeney LA, Rossi C, Kortmann BB, Devruyne FM, de la Rosette JJ: Long-term followup of randomized transurethral microwave thermotherapy versus transurethral prostatic resection. J Urol. 2001; 165: 1533-8.

38. Wagrell L, Schelin S, Nordling J, Richthoff J, Magnusson B, Schain M, et al.: Feedback microwave thermotherapy versus TURP for clinical BPH-a randomized controlled multicenter study. Urology 2002; 60: 292-9.

39. Arai Y, Aoki Y, Okubo K: Impact of interventional therapy for benign prostatic hyperplasia on quality of life and sexual function: a prospective study. J Urol. 2000; 164: 1206-11.

40. Djavan B, Seitz C, Ghawidel K, Basharkhah A, Bursa B, Hruby S, et al.: High-energy randomized transurethral microwave thermotherapy in patients with acute urinary retention due to benign prostatic hypertrophy. Urology 1999; 54: 18-22.

41. Floratos DL, Sonke GS, Francisca EA: High energy transurethral microwave thermotherapy for the treatment of patients in urinary retention. J Urol. 2000; 163: 1457-60.

42. Schelin S: Microwave thermotherapy in patients with benign prostatic hyperplasia and chronic urinary retention. Eur Urol. 2001; 39: 400-4.

43. Francisca EA, d'Ancona FC, Meuleman EJ, Debruyne FM, de la Rosette JJ: Sexual function following high energy microwave thermotherapy: Results of a 
randomized controlled study comparing transurethral microwave thermotherapy to transurethral prostatic resection. J Urol. 1999; 161: 486-90.

44. Norby B, Frimodt-Moller PC: Development of a urethrorectal fistula after transurethral microwave thermotherapy for benign prostatic hyperplasia. BJU Int. 2000; 85: 554-5.
45. Lin DC, Lin TM, Tong YC: Emphysematous prostatic abscess after transurethral microwave thermotherapy. J Urol. 2001; 166: 625.

46. Liatsikos EN, Dinlenc CZ, Kapoor R, Smith AD: Transurethral microwave thermotherapy for the treatment of prostatitis. J Endourol. 2000; 14: 68992.

Received: November 8, 2002 Accepted: December 5, 2002

$\overline{\text { Correspondence address: }}$

Dr. Kevin T. McVary

Dept Urology, Feinberg Medical School

Northwestern University, Tarry 11-715

300 E Superior St

Chicago, Illinois, 60611, USA

Fax: + 312 908-7275

E-mail:k-mcvary@northwestern.edu 